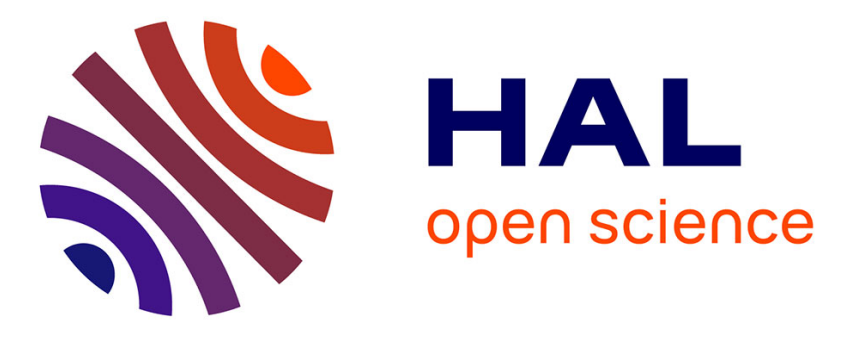

\title{
Unsupervised Fiber Bundles Registration using Weighted Measures Geometric Demons
}

Viviana Siless, Sergio Medina, Pierre Fillard, Bertrand Thirion

\section{To cite this version:}

Viviana Siless, Sergio Medina, Pierre Fillard, Bertrand Thirion. Unsupervised Fiber Bundles Registration using Weighted Measures Geometric Demons. Workshop on Multi Modal Brain Image Analysis, Li Shen - Tianming Liu - Pew-Thian Yap - Heng Huang - Dinggang Shen -Carl-Fredrik Westin, Sep 2013, Nagoya, Japan. hal-00853582

\section{HAL Id: hal-00853582 https://hal.inria.fr/hal-00853582}

Submitted on 22 Aug 2013

HAL is a multi-disciplinary open access archive for the deposit and dissemination of scientific research documents, whether they are published or not. The documents may come from teaching and research institutions in France or abroad, or from public or private research centers.
L'archive ouverte pluridisciplinaire HAL, est destinée au dépôt et à la diffusion de documents scientifiques de niveau recherche, publiés ou non, émanant des établissements d'enseignement et de recherche français ou étrangers, des laboratoires publics ou privés. 


\title{
Unsupervised Fiber Bundles Registration using Weighted Measures Geometric Demons
}

\author{
Viviana Siless $^{1,2}$, Sergio Medina ${ }^{1,2}$, Pierre Fillard $^{1}$, and Bertrand Thirion ${ }^{1,2}$ \\ 1 Parietal Team, Inria Saclay-Île-de-France, Saclay, France, \\ viviana.siless@inria.fr, \\ http://parietal.saclay.inria.fr \\ 2 CEA, DSV, I²BM, Neurospin bât 145, 91191 Gif-Sur-Yvette, France
}

\begin{abstract}
Brain image registration aims at reducing anatomical variability across subjects to create a common space for group analysis. Multi-modal approaches intend to minimize cortex shape variations along with internal structures, such as fiber bundles. A difficulty is that it requires a prior identification of these structures, which remains a challenging task in the absence of a complete reference atlas. We propose an extension of the log-Geometric Demons for jointly registering images and fiber bundles without the need of point or fiber correspondences. By representing fiber bundles as Weighted Measures we can register subjects with different numbers of fiber bundles. The efficacy of our algorithm is demonstrated by registering simultaneously $T_{1}$ images and between 37 and 88 fiber bundles depending on each of the ten subject used. We compare results with a multi-modal $T_{1}+$ Fractional Anisotropy (FA) and a tensor-based registration algorithms and obtain superior performance with our approach.
\end{abstract}

Keywords: Registration, neural fibers, diffeomorphism, Demons Algorithm, multi-modal registration, image, geometry, log-domain.

\section{Introduction}

In medical image analysis, non-linear image registration intends to reduce anatomical variability across subjects in order to ease subsequent subjects or population comparisons. Over the last decades the availability of different image modalities has increased, bringing hope for more accurate registration procedures. $T_{1}$ weighted image ( $T_{1}$ image in the sequel) registration is mainly driven by the contrast of the the grey matter and ventricles. In these images, the white matter appears uniform, giving no relevant information about its internal structures, which are composed of neural fibers connecting cortical areas. However Diffusion Tensor Imaging (DTI) can be used to reveal the microscopic structure of the white matter. Aligning white matter structures can help to increase the sensitivity of fMRI activation detection as shown in [1] and in 4] a group analysis on DWI was performed for early detection of schizophrenia. Tensor-based registration has recently been proposed to improve white matter alignment [14]13]. 
Nevertheless, mis-registration may persist in regions where the tensor field appears uniform [5].

Multi-modal registration combines information from different image modalities to provide more anatomical details. For instance, the registration algorithm in [3] uses $T_{1}$ images and FA from DTI to better align grey and white matter. Geometric registration specifically targets the alignment of Structures of Interest (SOIs), such as in [12 for cortical surfaces, or 5 for fiber bundles. While those clearly improve SOI registration, they may not be suitable for aligning other structures than those used specifically during registration.

Hybrid techniques propose to jointly consider SOIs and images during registration. For instance, in 277 the mathematical framework of Measures and Currents respectively, were used to simultaneously register images and geometric descriptors such as sulcal lines or surfaces, while [9] proposed a Markovian solution to the same problem.

In the log-Geometric Demons with Currents (CGD) 8 an hybrid multimodal registration of iconic and geometric descriptors has been proposed that uses Currents to model fibers but relies on a one-to-one fiber bundles correspondences across subjects. Correspondences across subjects are hard or even impossible to obtain. Individual fiber bundles show important differences in compactness, length and density. These characteristics might depend on the subject, tractography parameters and on the quality of the images. Furthermore, in some cases bundles may be cut, fused or absent in some subjects.

We propose to represent our geometric descriptors of fiber bundles as Weighted Measures to relax the hypothesis of explicit fiber correspondences across subjects. We define a flexible framework in which subjects can have different number of bundles, and no assumption is made about the bundle size.

The rest of the paper is organized as follows. First, we propose a mathematically sound extension of the log-Geometric Demons, the Weighted Measures Geometric Demons (WMGD), that relies on the log-domain daemons framework for computation purposes and handles geometric constraints as Weighted Measures. Then, we evaluate the WMGD $T 1+$ bundles constraints registration on a dataset of 10 subjects and compare them with a tensor-based [13, and ANTS 3 a $T_{1}+$ FA multi-modal registration. We also study the sensitivity of the results with respect to the various parameters.

\section{Weighted Measures Geometric Demons (WMGD)}

WMGD is a multi-modal algorithm that jointly registers image and geometric descriptors. We shortly describe in Section 2.1 the $T_{1}$ diffeomorphic demons registration, for then explain in Section 2.2 the extension to the geometric registration and the modeling of our constraints as Weighted Measures.

\subsection{Image Registration: The Diffeomorphic Demons}

The goal of image registration is to find the displacement field $s$ that aligns as accurately as possible the corresponding structures from a moving image $M$, to 
the structures in the fixed image $F$. Ideally the displacement field $s$ minimizes a distance between the fixed and the moving image, while holding some properties such as being diffeomorphic.

In the Demons framework 10 a correspondence field $c$ was introduced to make the minimization of the functional energy tractable: $E(c, s)=\frac{1}{\sigma_{i}^{2}} \operatorname{Sim}(F, M \circ$ $c)+\frac{1}{\sigma_{x}^{2}} \operatorname{dist}(s, c)^{2}+\frac{1}{\sigma_{T}^{2}} \operatorname{Reg}(s)$, where Sim is a similarity measure between images defined by the sum of square differences (SSD) and $\operatorname{Reg}(s)$ a regularization term chosen to be the harmonic energy $\|\nabla s\|^{2}$. The amount of regularization is controlled with $\sigma_{T}$ while $\sigma_{i}$ accounts for the image noise.

The term dist $(s, c)^{2}$ imposes the displacement field $s$ to be close to the correspondence field $c$. And $\sigma_{x}$ weights the spatial uncertainty on the deformation. The energy minimization is performed by alternating minimization w.r.t. $c$ and $s$. In [11, small deformations are parametrized by a dense displacement field $u$ : $c \leftarrow s$ exp $(u), \exp ()$ being the exponential map in the Lie group sense, which ensures that the result is diffeomorphic. In the log-domain demons $s$ is encoded with the exponential map as $s=\exp (v)$ and the inverse of $s$ can be easily computed as $s^{-1}=\exp (-v)$; then $\operatorname{dist}(s, c)=\left\|\log \left(s^{-1} \circ c\right)\right\|$ and $\operatorname{Reg}(s)=\|\nabla \log (s)\|^{2}$ where $\log =\exp ^{-1}$.

\section{$2.2 \quad T_{1}+$ Geometric Registration}

We build on the extension of the Demons framework proposed in [8, that includes geometric descriptors into the variational formulation. The definition of $c$ carries information coming from both image and geometry. Let $\mathcal{G}^{F}$ be the fixed geometric descriptors and $\mathcal{G}^{M}$ the moving one, we aim at minimizing the following energy:

$$
\begin{aligned}
E(c, s)= & \frac{1}{\sigma_{i}^{2}}\left[\operatorname{Sim}_{I}(F, M \circ c)+\operatorname{Sim}_{G}\left(c \star \mathcal{G}^{F}, \mathcal{G}^{M}\right)\right]+ \\
& \frac{1}{\sigma_{x}^{2}} \operatorname{dist}(s, c)^{2}+\frac{1}{\sigma_{T}} \operatorname{Reg}(s),
\end{aligned}
$$

where $\operatorname{Sim}_{I}$ is the image similarity criterion, $\operatorname{Sim}_{G}$ the geometric similarity criterion, and $c \star \mathcal{G}^{F}$ denotes the action of $c$ on the geometry. Then $c$ is parametrized by an update field of image and geometry which is described at the end of this section. Note that $s$ goes from $F$ to $M$, thus the inverse of $s$ gives the geometric deformation.

\section{$2.3 \quad$ Fiber Bundles Representation}

In the Currents GD fiber bundles were represented in the space of currents as it provides a pose and shape-sensitive measure, independent of the number of fibers per bundle. The main issue with this metric is the need for corresponding bundles, hence requiring prior identification. Currents could in theory be used to represent a set of geometric objects without explicit correspondences, but they 
require an orientation to be chosen for each fiber: given a curve $L$ and a sequence of points $L=x_{1}, \ldots, x_{n}$ a current is defined as $\sum_{i} \tau_{i} \delta_{c_{i}}$, where $c_{i}=\frac{x_{i}+x_{i+1}}{2}, \tau_{i}=$ $x_{i}-x_{i+1}$, in other words, a set of positions and tangent vectors. A current can thus be seen as a sum of oriented segments. Therefore it is important to find a consistent orientation, otherwise the same fiber with the opposite orientation cannot be registered properly. It is extremely hard to find a consistent orientation on a large number of one-dimensional objects in $3 \mathrm{D}$ without a prior segmentation and labeling in each subject. As subject variability is high and -in the absence of complete fiber atlas- correspondence mistakes can lead to poor solutions, we propose to relax this hypothesis and represent the geometry using Weighted Measures.

\subsection{Compression of the tractography output}

Depending on the resolution of the diffusion images the number of fibers can go from few thousands to few millions, generally leading to high computational cost. For this reason we want to reduce the fibers to a set of few representatives, and give these representatives a weight corresponding to the number of fibers that they represent. For registration purposes we need bundles to be highly homogeneous so that each representative summarizes the bundle accurately. To obtain low variance bundles we require many of them (typically 500) among which the small ones are considered as outliers and discarded.

For registration we take the largest (i.e. more than 50 fibers) and the longest ones (i.e. more than $50 \mathrm{~mm}$ ) as we believe they can better lead the alignment of the white matter. Ideally this yields to 50-100 fiber bundles. Large bundles have higher probability of being well defined, hence detected across subjects. Short fibers, specifically U-shape fibers are accumulated around the cortex and it is hard to distinguish one from the others because of their resemblance on position and shape. In consequence, they can easily mislead the registration close to the cortical foldings.

\subsection{Weighted Measures}

Having a set of bundles from the fixed fibers $C^{F}$ (and moving $C^{M}$ ), we define the set of points in $\mathcal{G}^{F}$ as $x_{i, j}, i \in\left[1 . .\left|C^{F}\right|\right], j \in\left[1 . .\left|C_{i}^{F}\right|\right]$, where $x_{i, j}$ the jth point of the representative from the i-th bundle in $C^{F}$. We can associate with this sequence a specific measure as a sum of weighted Dirac Measures: $\mu_{\mathcal{G}}^{F}=\sum_{i=1}^{\left|C^{F}\right|} \sum_{j=1}^{\left|C_{i}^{F}\right|} w_{i, j} \delta_{x_{i}}$ where $w_{i, j}=\frac{\left|C_{i}^{F}\right|}{\sum_{i=0}^{\left|C^{F}\right|}\left|C_{i}^{F}\right|}$, which weights measures according to the number of fibers that the bundle of $C_{i}^{F}$ represents. From now on, to simplify notations, we refer to points from the geometry in $\mathcal{G}^{F}$ as $x_{i}$ and to points from $\mathcal{G}^{M}$ as $y_{i}$, and we assume that $w_{i}^{x}$ (resp. $w_{i}^{y}$ ) is the weight of the $x_{i}$ (resp. $y_{i}$ ) given by the corresponding bundle size. We define the geometry in the fixed subject as $\mathcal{G}^{F}=\sum_{i=0}^{N} w_{i}^{x} \delta_{x_{i}}$ and for the moving subject as $\mathcal{G}^{M}=$ $\sum_{j=0}^{M} w_{j}^{y} \delta_{y_{j}}$. 
Let $\mathcal{G}^{F}=\left(x_{1}, \ldots, x_{N}\right)$ and $\mathcal{G}^{M}=\left(y_{1}, \ldots, y_{M}\right)$ be the fixed and a moving geometric descriptor and $N, M$ being the number of objects. Let $K_{\beta}$ be a Gaussian kernel of size $\beta$. Then the scalar product between two sums of Weighed Measures can be expressed conveniently with a pre-defined kernel $K_{\beta}$ :

$$
\left\langle\mathcal{G}^{F}, \mathcal{G}^{M}\right\rangle_{\beta}=\left\langle\sum_{i=1}^{N} w_{i}^{x} \delta_{x_{i}}, \sum_{j=1}^{M} w_{j}^{y} \delta_{y_{j}}\right\rangle_{\beta}=\sum_{i=1}^{N} \sum_{j=1}^{M} K_{\beta}\left(x_{i}, y_{j}\right) w_{i}^{x} \cdot w_{j}^{y}
$$

Then the distance between Weighted Measures is defined as follows:

$$
d_{\beta}^{2}\left(\mathcal{G}^{F}, \mathcal{G}^{M}\right)=\left\|\mathcal{G}^{F}\right\|^{2}+\left\|\mathcal{G}^{M}\right\|^{2}-2\left\langle\mathcal{G}^{F}, \mathcal{G}^{M}\right\rangle_{\beta}
$$

The distance captures misalignment and shape dissimilarities at the resolution $\beta$. Distances much larger than $\beta$ do not influence the metric, while smaller ones are considered as noise and thanks to the smoothing effect of the kernel they are not taken into account.

Given the current deformation $s$, we define the action of the correspondence field $c$ on $\mathcal{G}$ as: $\left.c \star \mathcal{G}=\left\{s \circ \exp \left(u_{G}\right)\left(x_{i}\right)\right\}_{i \in[1, N]} \approx s\left(x_{i}\right)+u_{G}\left(s\left(x_{i}\right)\right)\right\}_{i \in[1, N]}$.

Since we deal with a discrete set of points, we choose to parametrize the dense update field $u_{G}$ by a finite set of vectors $u_{G, i}$ using radial basis function extrapolation: $u_{G}(x)=\sum_{i=1}^{N} h\left(\left\|x-x_{i}\right\|\right) \lambda_{i}$, where $h(x)=e^{-\frac{x^{2}}{\gamma^{2}}}, \lambda_{i}$ are the interpolation coefficients and $\gamma>0$ is the interpolation scale. $\lambda_{i}$ are calculated such that $u_{G}\left(x_{i}\right)=u_{G, i} \forall i$. Let us define the matrix $A$ such that $[A]_{i, j}=h\left(\| x_{i}-\right.$ $\left.x_{j} \|\right)\left([A]_{i, j}\right.$ denotes the $(i, j)$ entry of $\left.A\right), \Lambda=\left[\lambda_{1}, \ldots, \lambda_{N}\right]$ the vector of $\lambda \mathrm{s}, H(x)$ the vector such that $[H(x)]_{i}=h\left(\left\|x-x_{i}\right\|\right)$ and $U=\left[u_{G, 1}, \ldots, u_{G, N}\right]$. We can write: $u_{G}(x)=H(x) A^{-1} U$. Minimizing $\nabla E_{G}\left(s, u_{G}\right)=0$ w.r.t. $u_{G}$ via gradient descent yields to the following update field equation:

$$
\begin{aligned}
u_{G, i}= & -\epsilon\left[-\frac{2}{\beta^{2}} \sum_{l=0}^{N} w_{l}^{x} w_{i}^{x} K_{\beta}\left(s\left(x_{i}\right), s\left(x_{l}\right)\right)\left(s\left(x_{i}\right)-s\left(x_{l}\right)\right)\right. \\
& \left.+\frac{2}{\beta^{2}} \sum_{j=0}^{M} w_{i}^{x} w_{j}^{y} K_{\beta}\left(s\left(x_{i}\right), y_{j}\right)\left(s\left(x_{i}\right)-y_{j}\right)\right],
\end{aligned}
$$

where $\epsilon \in[0,1]$, and $\gamma \in[1,4] \mathrm{mm}$.

Finally, a new update field is defined by the additive combination of the image update field $u_{I}$ described in [11, and the geometric update field $u_{G}$ in eq. (4): $u=u_{I}+u_{G}$. The algorithm follows as in [1] where a regularization is applied and the transformation $s$ is updated. In 8] non-intersecting domains where assumed between the image and the geometry of the fibers. However, this contradicts the minimization procedure, thus we do not use such a splitting here.

\section{Joint $T_{1}$ MRI and Brain Bundle Registration}

In this section we describe the experiments of the extended joint $T_{1}$ MRI and brain fibers bundles registration. We first analyse the behaviour of the method itself, and we later compare it against other methods. 


\subsection{Data Description}

We used ten healthy volunteers from the Imagen database scanned with a $3 \mathrm{~T}$ Siemens Tim Trio scanner acquisitions were MPRAGE for $T_{1}$ weighted ( $240 \times$ $256 \times 160,1.09375 \times 1.09375 \times 1.1 \mathrm{~mm})$ and DW-MRI $(128 \times 128 \times 60,2.4 \times 2.4 \times$ $\left.2.4 \mathrm{~mm}^{3}\right) \mathrm{TR}=15000 \mathrm{~ms}, \mathrm{TE}=104 \mathrm{~ms}$, flip angle $=90^{\circ}, 36$ gradient directions, and $\mathrm{b}$-value $=1300 \mathrm{~s} / \mathrm{mm}^{2}$.

For each subject we obtained the linear transformation from the non-weighted image $B=0$ to $T_{1}$ to align bundles with $T_{1}$ images. Eddy currents correction were applied to DTI data, and skull and neck were removed from $T_{1}$ images using the FSL software.

We used MedInria for fiber tractography, and splines to extrapolate for uniformly distributed points. Fibers shorter than $50 \mathrm{~mm}$ were discarded in order to discard U-shape fibers. Within U-shape brain fibers variability is high and mismatch across subjects can be easily introduced during registration. We rather trust the image for those regions around the cortex.

As discussed in section 2.4 we are not interested in using the whole fiber tracktography output, but rather the bundles representatives. We use QuickBundles algorithm [6] to obtain a clustering of the fibers, and used the representatives given by the algorithm for registration. The threshold value for the bundles spread width was set to $10 \mathrm{~mm}$, which gives a trade-off between low cluster variability and number of fibers per cluster. This yielded an average of 600 bundles per subject (range: [323,927]), where a bundle contains at least one fiber. Selecting bundles with more than 50 fibers leads to an average of 63 bundles (range: $[37,88])$. In the sequel we refer to this subset as the Training Set, and bundles with less than 50 fibers as the Test Set.

Before registering with WMGD we apply a $T_{1}$ affine transformation using the MedInria software to take subjects to the target space, and apply the inverse to the fibers.

After running WMGD we obtained a deformation field that we applied to the moving $T_{1}$ and the inverse of the deformation to the fibers.

The rest of this section explains the experiments performed. To assess the sensitivity to parameters we arbitrary choose a subject as target to register the rest of the dataset. Then, for performance comparison with other algorithms, we register the subjects to one another, and average the pairwise distances.

\subsection{Weighted Measures kernel size}

To analyze the impact the $\beta$ parameter we register the whole dataset to an arbitrary subject chosen as target by using the Training Set of bundles. Typically large kernels would be able to capture large misalignment and handle details as noise.

We run Weighted Measures Geometric Demons with the following parameters fixed $\epsilon=0.3, \gamma=3 \mathrm{~mm}, \sigma_{T}=2, \sigma_{i}=1, \sigma_{x}=1$ with a 3 -steps multi-scale approach with 15,10 and 5 iterations at each scale (from small to large). 
We vary $\beta$ from 0 to $30 \mathrm{~mm}$ and show results in Figure 1 for fibers (a) and for image (b). However, we believe that decreasing $\beta$ through iterations might avoid local minima for fiber registration and improve the accuracy of image registration. We decrease it $0.5 \%$ at each iteration and results are shown in Figure 1 for fibers (c) and for image (d).

\subsection{Regularization}

To analyze the smoothness of the deformation field, we run experiments with values of $\sigma_{T}$ varying from 0 to 3.0 and analyze the impact over the results. Analogous to Section 3.2 we register the dataset to an arbitrary subject chosen as target, and we use the Training Set of fibers for registration. We use the same parameter setting with $\beta=20 \mathrm{~mm}$. In Figure 2 results are shown for fibers (a), image (b) and the regularization term (c).

\subsection{Performance comparison}

We conducted a cross-validation experiment by performing registration on the Training Set, and validating results over the Test Set (bundles left out for containing less than 50 fibers). To validate the robustness of the results we register subjects dataset to one another and plot the average pairwise distance.

We run Weighted Measures Geometric Demons with the following parameters $\epsilon=0.3, \gamma=3 \mathrm{~mm}, \beta=10 \mathrm{~mm}, \sigma_{T}=2, \sigma_{i}=1, \sigma_{x}=1$. $\beta$ was decreased by $0.5 \%$ at each iteration of the algorithm. Symmetric Tensor Demons (STD) was run with its defaults parameters. For ANTS we used recommended parameters from documentation except for the weight of $T_{1}$ and $F A$ where different combinations were tested. We finally show results with both equal to 1 , which we found to be a fair trade-off. Each algorithm was tested on the 3-steps multi-scale approach with 15,10 and 5 iterations at each scale (from small to large).

We recall that for WMGD we first apply a $T_{1}$ affine transformation using the MedInria software to take subjects to the target space, and apply the inverse to the fibers. After running WMGD we obtained a deformation field that we applied to the moving $T_{1}$ and the inverse of the deformation to the fibers. We compare our results to those of ANTS, a multi-modal image registration combining $T_{1}$ image and FA, and Symmetric Tensor Demons, a tensor-based registration algorithm. Before running ANTS, corresponding $\mathrm{FA}$ and $T_{1}$ were aligned using a linear transformation. The resulting affine transformation and deformation field were applied to the moving $T_{1}$ image, and their inverse to the fibers. For tensor-based registration, tensor images were taken to the target $T_{1}$ space using an affine transformation for the moving subjects, but preserving original resolution. The resulting deformation field was up-sampled to the $T_{1}$ resolution for application to the image and then inverted for application to the geometry. 


\section{Results and Discussion}

\subsection{Weighted Measure: $\beta$ value}

As expected, Figure 1 shows that higher $\beta$ values give better scores for fiber registration, while loosing accuracy on the image registration. Each curve in Figure 1 has been normalized by its maximum value in order to analyze the impact of the parameter in each subject. However minimum values across figures are not comparable as they depend on the maximum value achieved. When defining a fixed $\beta$ though the iteration we quickly lose accuracy for the image registration. When decreasing iteratively $\beta$, we can see that with an initial values between 10 and 15, we improve fiber alignment while still holding the image one.

\subsection{Regularization}

In Figure 2 we see that as we increase the regularization, the image accuracy decreases. However, low regularization will result in sharp deformations, which are often undesirable for the purposes of registration. As for the fiber accuracy we find the impact is low, nevertheless, a fair compromise with the harmonic energy can be found for $\sigma_{T}$ between 1.5 and 2 . The difference of regularization impact over the image and the fibers are related to the resolution differences.

\subsection{Performance Comparison}

The aim of WMGD is to align $T_{1}$ images and neural fibers simultaneously by only using a set of bundles that represent well the white matter structure. We compare our results to a tensor-based registration (Symmetric Tensor Demons) and a multi-modal registration of $T_{1}+\mathrm{FA}$ (ANTS).

Average results for registering the individual datasets to each other are shown in Fig. 3 for training set (a), test set (b) and image (c). The WMGD method outperforms the others on the bundles used in the registration as shown in (a), which is expected, given that the minimized energy considered those specific bundles. For a fair comparison we tested our metric on the remaining bundles; the corresponding results are shown in (b). For the left aside bundles, results are similar but generally improved by our method. These results suggest that a sparse bundle selection according to their importance can be sufficient and that there is no need to require datasets to have the same number of bundles. Last, in (c) we compare the methods with respect to image registration accuracy. It is important to mention that diffusion images had a lower resolution than the $T_{1}$ images, giving advantage to ANTS and our algorithm in accuracy. WMGD yields better performance than ANTS, proving that improvements on bundles registration was not obtained at the expense of image accuracy.

In Fig. 4 we can see the result of registering the dataset to an arbitrary chosen as target. In (d) we see the fibers even before applying an affine registration, and in (g) we can see some improvements with respect to (e) and (f) regarding the borders of the image, and a better alignment of the corpus callosum. 


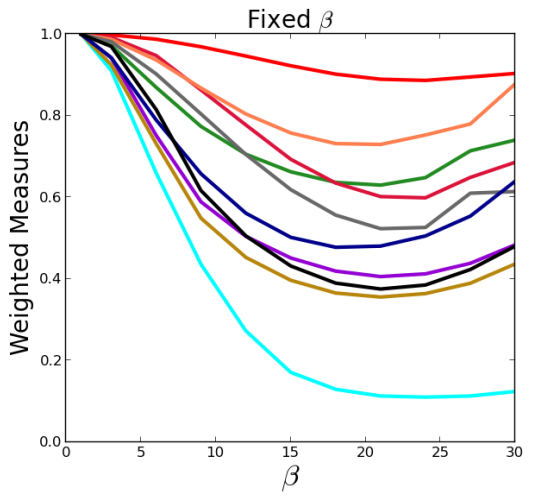

(a)

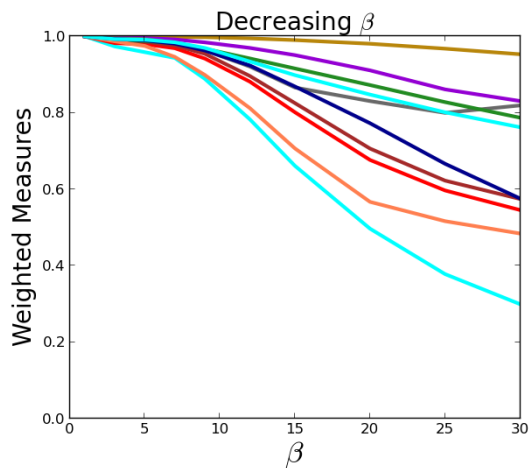

(c)

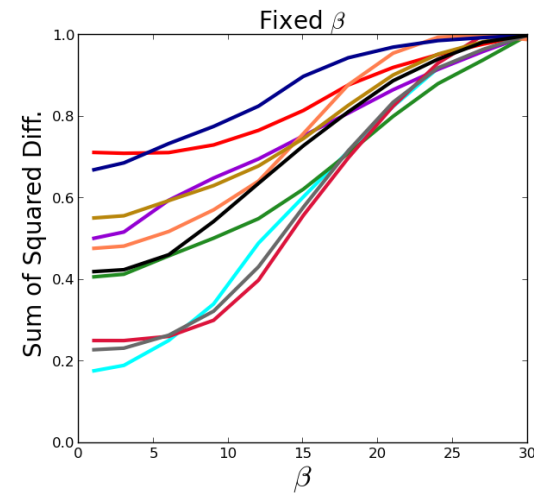

(b)

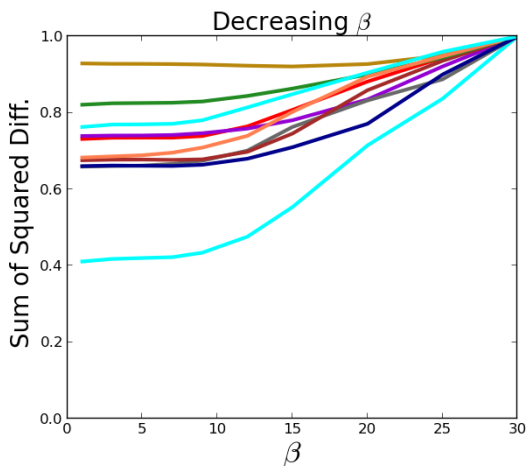

(d)

Fig. 1. Registration of the dataset to an arbitrary subject chosen as target. Each curve encodes one subject registration to the target. $\beta$ varies in the $\mathrm{x}$-axis. Figures (a) and (b) show the metric for fiber and image respectively at each $\beta$ value fixed through iterations. Figures (c) and (d) show respectively the metric for fibers and image at each initial value of $\beta$, and with a $0.5 \%$ decrease at each iteration. Curves were normalized by their maximal value. 


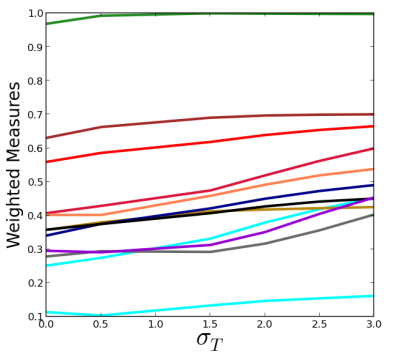

(a)

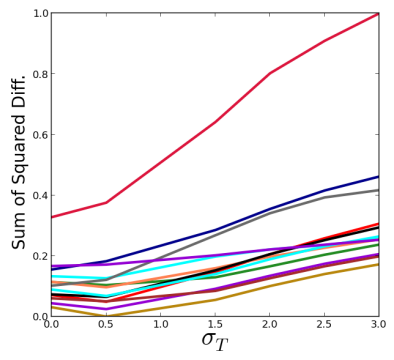

(b)

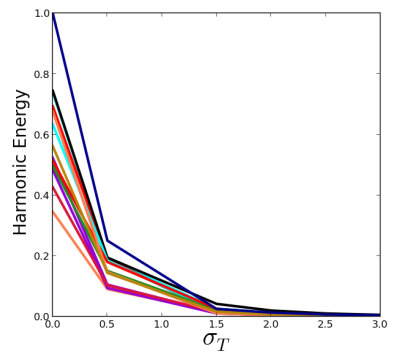

(c)

Fig. 2. Registration of the dataset to an arbitrary chosen as target with varying $\sigma_{T}$. Each curve encodes one subject registration to the target. Weighted Measures metric is shown in (a) for increasing $\sigma_{T}$, Sum of Squared Diff. of the image in (b) and in (c) we show the harmonic energy results. Curves were scaled using min-max normalization.

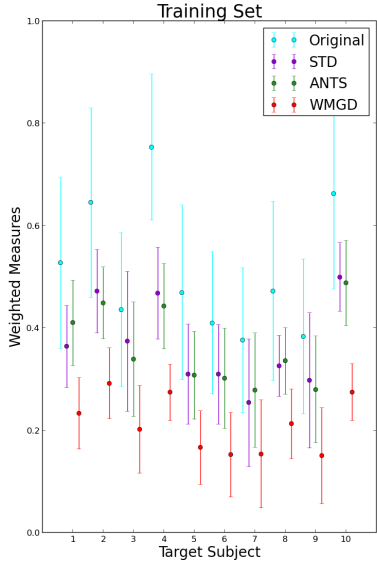

(a)

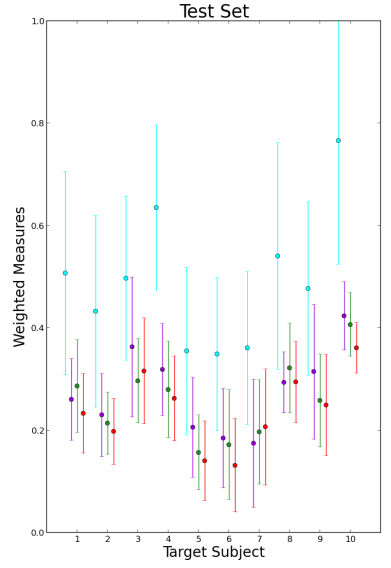

(b)

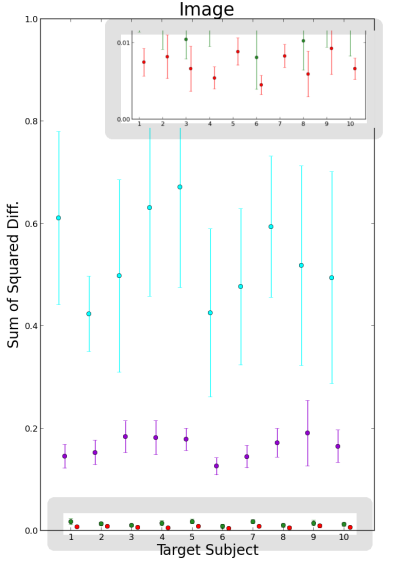

(c)

Fig. 3. Registration of the dataset to each subject. STD, ANTS, and WMGD show the average accuracy of the registration to each subject for the corresponding method. Original corresponds to the original distances between the dataset and the chosen target subject. Values were scaled using min-max normalization. 


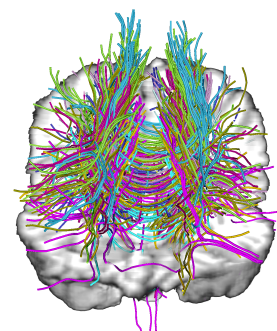

(d) Original

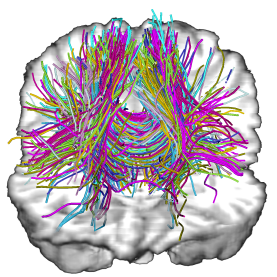

(e) STD

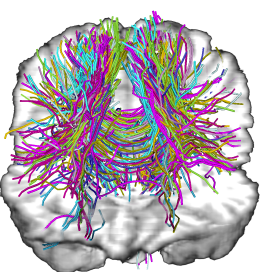

(f) ANTS

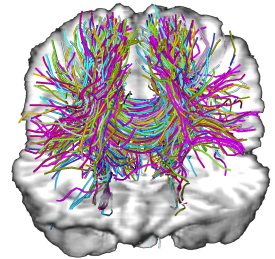

(g) WMGD

Fig. 4. Overlapping of fiber bundle representatives from all subjects registered to an arbitrary one chosen as target. Colors encode the different subjects. Behind we see the $3 D T_{1}$ image of the target subject.

We time all algorithms with an Intel Xeon 8proc. $2.53 \mathrm{GHz}, 11.8 \mathrm{~Gb}$ and obtained: $\mathrm{STD}=5.11 \mathrm{~min}, \mathrm{Ants}=29.43 \mathrm{~min}, \mathrm{WMGD}=44.0 \mathrm{~min}$.

\section{Conclusion}

We presented a novel approach of hybrid multi-modal registration based on $T_{1}$ images and representative fiber bundles. Our algorithm does not require to have the same number of fiber bundles per subject, neither does it require them to be oriented, which makes it usable in much more general and realistic situations than previous approaches.

We have compared our algorithm to other well-known available registration algorithms with different modalities $\left(T_{1}+\mathrm{FA}\right.$ and Tensors) to show the benefits of using geometric descriptors. We obtain very good results for training fiber bundles, and improvements on test fibers and image alignment can be seen.

We also show that using fiber bundles instead of tensor information or FA adds relevant features, which amounts to include some priors: Tractography algorithms have to deal with uncertain regions on DTI images and use prior knowledge to overcome this difficulties. We strongly believe that this information should not be discarded. Also, keeping only long fibers introduces prior knowledge into the registration process.

Future extensions of the present work could include a joint labeling/registration framework. One can expect that including fiber classification across the iterations of the registration will improve accuracy and also give relevant information for posterior analyses of groups of subjects.

In addition, the high performance of the algorithm makes it worthwhile for validating results on diseases where the white matter is degenerated.

Since neural fibers contains information about connected regions, we also conjecture that this methodology will increase sensitivity in fMRI activation detection experiments. 
Acknowledgments. This work was supported by ANR grant (ANR-10-BLAN0128). The data were acquired within the Imagen project.

\section{References}

1. Ardekani, B.A., Bachman, A.H., Strother, S.C., Fujibayashi, Y., Yonekura, Y.: Impact of inter-subject image registration on group analysis of fmri data. ICS 1265(0), 49 - 59 (2004)

2. Auzias, G., Colliot, O., Glaunes, J., Perrot, M., Mangin, J.F., Trouve, A., Baillet, S.: Diffeomorphic brain registration under exhaustive sulcal constraints. IEEE Trans Med Imaging (Jan 2011)

3. Avants, B.B., Tustison, N.J., Song, G., Cook, P.A., Klein, A., Gee, J.C.: A reproducible evaluation of ants similarity metric performance in brain image registration. NeuroImage 54(3), 2033 - 2044 (2011)

4. DeLisi, L.E., Szulc, K.U., Bertisch, H., Majcher, M., Brown, K., Bappal, A., Branch, C.A., Ardekani, B.A.: Early detection of schizophrenia by diffusion weighted imaging. Psychiatry Research: Neuroimaging 148(1), 61 - 66 (2006)

5. Durrleman, S., Fillard, P., Pennec, X., Trouvé, A., Ayache, N.: Registration, atlas estimation and variability analysis of white matter fiber bundles modeled as currents. NeuroImage 55(3), 1073-1090 (2011)

6. Garyfallidis, E., Brett, M., Correia, M.M., Williams, G.B., Nimmo-Smith, I.: Quickbundles, a method for tractography simplification. Frontiers in Neuroscience 6(175) (2012)

7. Ha, L.K., Prastawa, M., Gerig, G., Gilmore, J.H., Silva, C.T., Joshi, S.C.: Image registration driven by combined probabilistic and geometric descriptors. In: MICCAI. pp. 602-609 (2010)

8. Siless, V., Glaunés, J., Guevara, P., Mangin, J.F., Poupon, C., Bihan, D., Thirion, B., Fillard, P.: Joint t1 and brain fiber log-demons registration using currents to model geometry. In: MICCAI 2012, LNCS, vol. 7511 (2012)

9. Sotiras, A., Ou, Y., Glocker, B., Davatzikos, C., Paragios, N.: Simultaneous geometric-iconic registration. In: MICCAI. vol. 13, pp. 676-683 (2010)

10. Thirion, J.P.: Image matching as a diffusion process: an analogy with Maxwell's demons. Medical Image Analysis 2(3), 243-260 (Sep 1998)

11. Vercauteren, T., , Pennec, X., Perchant, A., Ayache, N.: Symmetric Log-Domain Diffeomorphic Registration: A Demons-Based Approach. In: MICCAI, LNCS, vol. 5241, chap. 90, pp. 754-761 (2008)

12. Yeo, B., Sabuncu, M., Vercauteren, T., Ayache, N., Fischl, B., Golland, P.: Spherical demons: Fast diffeomorphic landmark-free surface registration. IEEE Trans Med Imaging 29(3), 650-668 (2010)

13. Yeo, B., Vercauteren, T., Fillard, P., Peyrat, J.M., Pennec, X., Golland, P., Ayache, N., Clatz, O.: Dt-refind: Diffusion tensor registration with exact finite-strain differential. IEEE Trans Med Imaging 28(12), 1914-1928 (2009)

14. Zhang, H., Yushkevich, P.A., Alexander, D.C., Gee, J.C.: Deformable registration of diffusion tensor mr images with explicit orientation optimization. Medical Image Analysis 10(5), 764-785 (2006) 